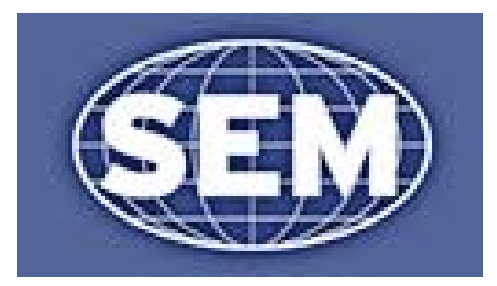

Article: "Comparison of Minimum Detectable Crack Size in a Geared System From Three Different Vibration Transducer Types"

Authors: R. Moreno, J.M. Chicharro and P. Pintado Experimental Techniques, Volume 38, Issue 1, January/February 2014, Pages: 76-87,

Key words:

Gear fault detection, laser vibrometer, accelerometer, microphone, transducer comparison 


\title{
Comparison of Minimum Detectable Crack Size in a Geared System From Three Different Vibration Transducer Types
}

\author{
Ricardo Moreno Sanchez* \\ Jose M. Chicharro ${ }^{\dagger}$ \\ Publio Pintado \\ Department of Mechanical Engineering, University of Castilla La Mancha \\ Ciudad Real, Spain 13071
}

\begin{abstract}
Acoustic and vibration signals contain distinctive patterns useful for detecting defects in machinery. A number of signal processing strategies has been proposed for detecting and quantifying incipient defects in gears. Non intrusive measurement techniques like those based on Laser Doppler Vibrometry and microphones need to be studied in order to help users in selecting the appropriate transducer according to the application. This paper presents an experimental design to determine, as a novel contribution, the minimum detectable crack size in a geared system comparing three different transducers: a microphone, an accelerometer and a laser Doppler vibrometer (LDV). The comparison intends to discriminate the three transducers with respect to its early detection capability. Experiments were conducted in a closed loop torque test rig for several torque levels and speeds. Experimental factorial design was used to determine the main
\end{abstract}

\footnotetext{
*e-mail:rmoreno@udea.edu.co Mechanical Engineering at University of Antioquia (Medellin, Colombia)

${ }^{\dagger}$ Corresponding author: Address:Department of Mechanical Engineering, University of Castilla La Mancha, Av Camilo Jose Cela s/n e-mail: josemanuel.chicharro@uclm.es

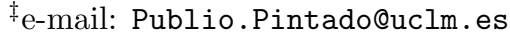




\section{Comparison of Minimum Detectable Crack Size in a Geared System From Three Different Vibration Transducer Types}

effects and their interactions in the detection process. The well-known Hilbert and wavelet transforms have been used as signal processing technique. Their advantages in the detection of incipient defects are highlighted in the paper. The results indicate that the acoustic signal stands out as the method that first detects an incipient progressive crack in gears (it detected cracks $1.3 \mathrm{~mm}$ long), although, as a drawback, the results obtained using the microphone signal are more sensitive to speed and torque. The second place was for LDV with $1.8 \mathrm{~mm}$ crack detection, and the third place for the accelerometer with $2.3 \mathrm{~mm}$ crack detection.

Keywords: Gear fault detection, laser vibrometer, accelerometer, microphone, transducer comparison

\section{Introduction}

Gears are widely used in machinery and in industrial applications. In some of these applications the gear represents a critical component since failure could result in a serious accident. The helicopter gearbox is probably the best example in this respect, and suffices to emphasize the reasons why the thrust for improving monitoring and diagnosis techniques has challenged academia and industry for decades.

There are mainly two mechanisms of crack initiation and growth in gear teeth. The first one is due to contact fatigue. Cracks initiate below the surfaces subjected to contact pressure (where the maximum shear stresses are found) and then grow to the surface following the major stress direction, thus producing small pits. The second mechanism is due to flexural fatigue at the tooth root where maximum tensile stresses (amplified by stress concentration) are found. This fatigue crack initiation may also be influenced by some of these factors: Moreno-Sanchez, Ricardo $\quad$ February 17, 2011 
Comparison of Minimum Detectable Crack Size in a Geared System From Three Different Vibration Transducer Types

deficient surface quality, low hardness, inadequate microstructure of the material or poor lubricating conditions [1].

A crack along the root of a tooth reduces the bending stiffness of the tooth increasing the elastic deflection [2]. This is perceived in the vibration spectrum as a change in amplitude as well as nonsymmetrical amplitude in the sidebands around gear mesh frequency, which is due to the disproportional clearance and impact energy [3]. Artificial defects introduced in the teeth by cutting its root up to a given depth reproduce this reduction of bending stiffness and, therefore, produce similar effects on the vibration spectrum as a crack.

An incipient crack in a spur wheel generates a low energy transient impulse that modulates the vibration signal as well as the acoustic emission. Some trends and patterns may be detected to infer the presence of the defect. These trends usually involve a small increase in the energy signal at rotational and mesh frequencies (and their harmonics) along with associated sidebands. A number of methods has been proposed for signal decomposition in order to extract information from the modulated signal and help detect the gear fault as early as possible.

Simple statistical parameters in the time domain are not suitable, by themselves, for detecting incipient (or even big) defects in low load systems ([4] and [5]). Mosher, Prior and Huff [6] found a complex interaction between torque, speed and the usual statistical parameters, that prevented detection and triggered numerous false alarms.

Fourier Transform (FT) can only detect faults in such an advanced state that the method is rendered useless since detection could almost be simultaneous to total failure. There are two main reasons why FT is not suitable for incipient fault detection: first, because the low energy impulses translate into imperceptible sidebands in the spectrum and, second, because

Moreno-Sanchez, Ricardo $\quad$ February 17, 2011 
Comparison of Minimum Detectable Crack Size in a Geared System From Three Different Vibration Transducer Types

FT is a highly non local transform, the time domain gets lost in the transformation, and the location of the defect cannot be inferred [7].

Short Time Fourier Transform (STFT) was proposed in order to overcome the high non locality of FT, but a big limitation still hinders this method: the window size is constant for all frequencies, so that different resolutions will be present in the spectrum.

Recent studies have tried to evaluate the suitability of other methods like Self-Similarity in vibration time series [8], iterated Hilbert transform [9], wavelet transform [10], neural networks [11]; and these come to join previous studies that tested the suitability of techniques such as Cepstral analysis, demodulation analysis, Wigner-Ville distribution [12] and autoregressive models [13]. There are several studies that emphasize the advantages of using Morlet wavelet for detecting faults in gears ([14] and [15]), whereas others recommend the Hilbert transform to identify the modulating effects for fault diagnosis [9]. Advanced and well documented signal processing techniques have been studied to give early detection of incipient cracks in gears. Among this large number of possible methods, we believe that the authors have been able to prove the synergic gains derived from the combined use of both the Hilbert and Wavelet transforms [16].

The comparison between results obtained using acoustic and vibration signals has also received the attention of researchers. A comparative study between acoustic and vibration signals, at three different torques, based on the analysis of root mean square (rms) for monitoring pitting in gears suggests a higher sensitivity of the acoustic signal [17]. Nevertheless, opposite results have been obtained in other studies of gear failure detection via vibration and acoustic signals using wavelet transform [18].

Similar comparisons between acoustic and vibration signals have been done for the case of Moreno-Sanchez, Ricardo

February 17, 2011 
detecting faults in bearings. A comparative study using rms and kurtosis, at different torques and velocities, suggests that acoustic emission provides earlier fault detection and improved identification capabilities [19]; while other studies conclude that accelerometers are better for detecting defects in bearings [20]. It should also be mentioned that some researchers have found difficulties for using simple statistical measurements (RMS, for example) with time domain acoustic signals [21].

Although each of the conclusions mentioned in the previous paragraphs are only valid when applied to the type of experiments from which they were obtained, the variety and discrepancies of results calls for a systematic experimental design. In other words, it is necessary to analyze the influence of torque and speed variations in the detection procedure for different transducers. One of these transducers, laser Doppler Vibrometer (LDV), has not been largely used for diagnostic purposes in geared systems and it is therefore interesting to include it in the comparisons.

Changes in factors like defect size, speed and torque and their effects on the acquired signal can be analyzed simultaneously by means of factorial experimental design. In this way, changes in the signal can be decomposed into contributions due to each factor having removed the effects of all others. Factorial designs have the advantages of being more efficient than one-factor-at-a-time experiments and they are necessary when interactions between factors may be present to avoid misleading conclusions. Moreover, factorial design allows to estimate the effect of each factor at several levels of the other factors, yielding conclusions that are valid over a range of experimental conditions [22].

During the 1990s many researchers begun using LDV in several applications, and in many cases the technique showed significant advantages over traditional contact sensors ([23], [24]).

Moreno-Sanchez, Ricardo $\quad$ February 17, 2011 
Comparison of Minimum Detectable Crack Size in a Geared System From Three Different Vibration Transducer Types

Accelerometers are intrusive in the sense that their mass is added to the system. On the other hand, LDV is a non intrusive technique (it performs a remote measurement), just like acoustic auscultation with a microphone, but, unlike the microphone, it is not affected by background noise. A systematic experimental design comparing LDV, microphone and accelerometer cannot yet be found in the literature.

The signal processing techniques used for detecting patterns take advantage from specific details in the signals, but since different transducers gather signals that have travelled through different paths, the "quality" and characteristics of said signals can differ dramatically. For example, whereas high frequencies (above $4 \mathrm{kHz}$ in our case) can travel with little disturbance to a microphone, they are highly damped out when using an accelerometer. It is this type of difference in behavior that motivates the multi-transducer comparison.

The work presented in this paper compares the diagnosis obtained using signals from three different transducers in a closed loop torque test rig. Analysis of variance (ANOVA) tests have been performed to determine the statistical significance of factors such as torque, rotational speed and defect size on the detection and quantifying processes. A statistics method based on evaluating the confidence band of least significant differences from the results of main effects in the factorial experiments makes it possible to compare the minimum detectable crack size.

\section{Experimental Set Up}

Figure 1 shows the test rig used for experiments. The working principles of the assembly have been used for this and other types of tests ([21], [25]). Four wheels meshed in two

Moreno-Sanchez, Ricardo

February 17, 2011 

Three Different Vibration Transducer Types

identical gear pairs can hold an inner closed loop torque when internal torsion is supplied. The shafts are actually made from two separate rods joined by central pairing flanges, so that a torque may be introduced in the system by twisting one flange with respect to its mating flange prior to joining them with screws. The relative phase angle in the coupling determines the torque, which can then be set at different levels. The loads on the contacting teeth on each gear can be very high, but the power demanded from the driving electric motor is only needed to overcome friction and dynamic loads. This is the main advantage of this system as compared to motor-gear-brake assemblies.

The test bench can be used with helical and spur gears. In this work, spur gears with 60 and 74 teeth were used. A torque range from 0 up to $150 \mathrm{Nm}$ is available, and the speed could be chosen in the range from 80 up to $1500 \mathrm{rpm}$.

The following devices were used for data acquisition: Polytec compact laser vibrometer (CLV-1000 laser module and CLV-700 sensor head), B\&K microphone type 4188(PCB amplifier, model 482A16) and B\&K accelerometer type 4371 (B\&K amplifier type Nexus 2692). National Instruments DAQCard 6062E. An SC-2345 Carrier with configurable connectors, and a 1000pulses/rev encoder. The sample frequency was $10 \mathrm{kHz}$ (A 5kHz lowpass Bessel filter was used to avoid aliasing). Table 1 shows sensitivities and frequency responses of transducers.

Unlike LDV, which can be pointed at a rotating surface, accelerometers must be placed on the bearing support because this is the fixed surface closest to the source of the vibrations of interest. But in its path from the meshing gears to the accelerometer, the waves pass through bearings and other elements where high frequency components are hindered [2] and the waves are, therefore, modulated.

Moreno-Sanchez, Ricardo $\quad$ February 17, 2011 
Comparison of Minimum Detectable Crack Size in a Geared System From Three Different Vibration Transducer Types

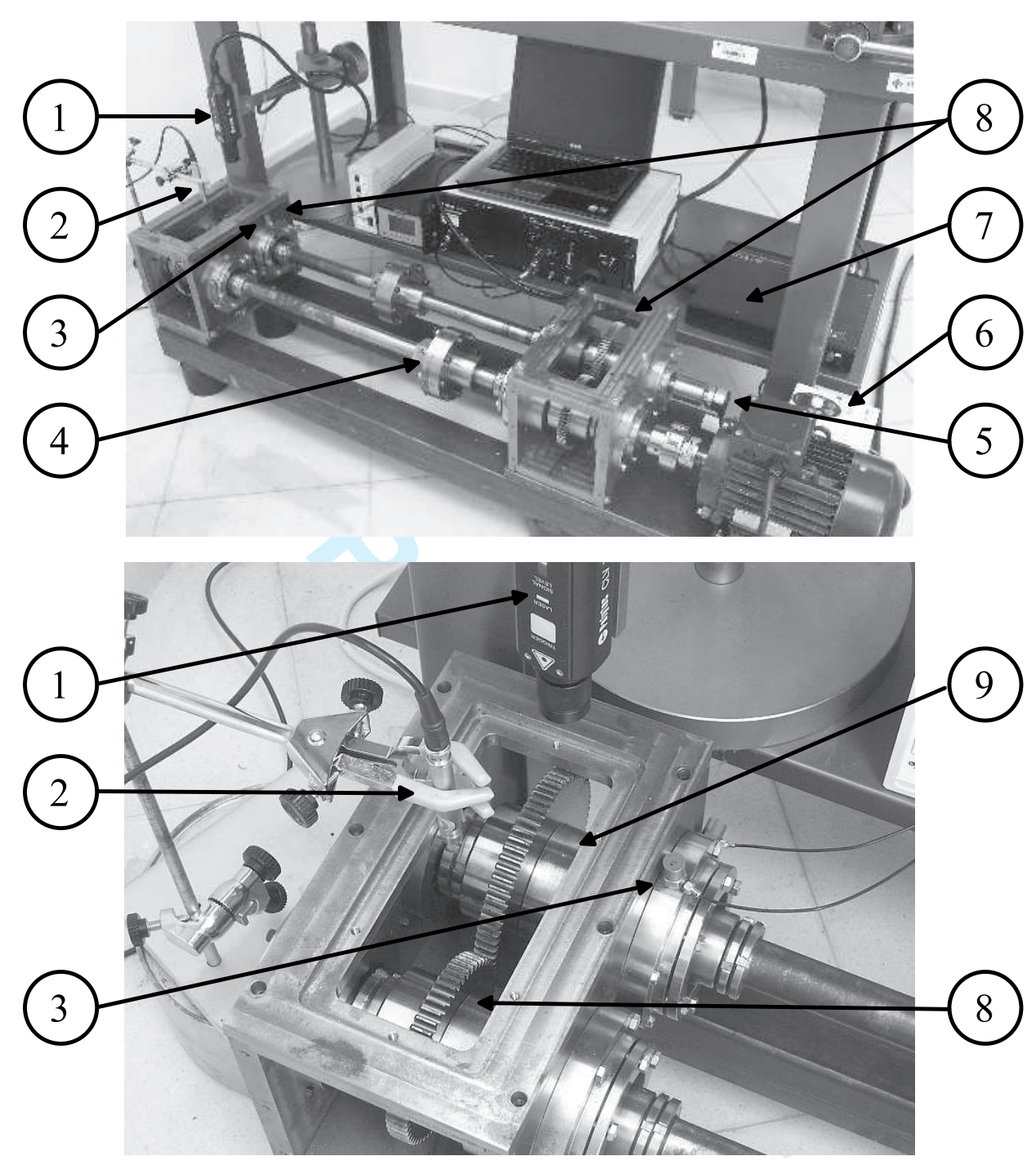

Figure 1: Test bench. 1-Laser Doppler vibrometer. 2-Microphone. 3-Accelerometer. 4Adjustable torque coupling. 5-Encoder. 6-Speed regulator. 7- Data acquisition hardware. 8- Spur gears. 9-Laser point location. 


\section{Comparison of Minimum Detectable Crack Size in a Geared System From} Three Different Vibration Transducer Types

Several researchers have made contributions on methodologies for optimum sensor placement. One of these methodologies is the effective independence method [26]. The method is based on the concept that, when possible, sensors should be placed in order to obtain mode shapes that are linearly independent of each other. In cases in which just one sensor is used, one should look for locations where the vibration is a result of the combination of the largest number of natural frequencies and modes.

Transducers must also be placed trying to optimize sensitivity over the expected frequency range. Several locations were evaluated to find a good position for accelerometers. Three accelerometers were located at axial, landscape and vertical directions (see Fig. 1) with the test bench at different operating ranges. Amplitudes and sensitivity where higher in the vertical direction, and this is why the transducers were placed to measure in this direction $[16]$.

LDV has the advantage over accelerometers that can be pointed at the surface of a rotating shaft. This is an advantage because the location is closer to the source of vibration and, therefore, not only the amplitudes of vibration are larger, but modulation of the signal when traveling through bearings is avoided, resulting in (one would expect) a much clearer

Table 1: Sensitivities and frequency responses of transducers

\begin{tabular}{|c|c|c|}
\hline SENSOR & SENSITIVITY & FREQUENCIES \\
\hline LDV & $0.5 \mathrm{~mm} / \mathrm{s}$ & $0.5 \mathrm{~Hz}-250 \mathrm{kHz}$ \\
\hline Accelerometer & $9.8 \pm 2 \% \mathrm{pC} / \mathrm{g}$ & $0.1 \mathrm{~Hz}-12.6 \mathrm{kHz}($ amplitude response $\pm 10 \%)$ \\
\hline Microphone & $31.6 \mathrm{mV} / \mathrm{Pa}$ & $8 \mathrm{~Hz}-12.5 \mathrm{kHz}( \pm 2 d B)$ \\
\hline
\end{tabular}

Moreno-Sanchez, Ricardo $\quad$ February 17, 2011 
Comparison of Minimum Detectable Crack Size in a Geared System From Three Different Vibration Transducer Types

signal to be processed. The fact that this location is not the same as that used for the accelerometer doesn't hinder the comparison between sensors, since the goal is to discriminate between them when they collect data at the best location possible and feasible for each type of transducer.

On the other hand, although shaft eccentricities and roughness will be "read" by the LDV, they do not interfere with the defect pattern recognition process mainly because they are typically small and constant for each rotation. The manufacturer of the LDV system used in this study describes the process for using LDV for measurements in rotating components. Tatar et al. [27] show that it is possible to use an LDV for measuring spindle dynamics when the surface is optically smooth.

The distance from the microphone to the meshing teeth is six centimeters. Lateral panels were removed to diminish interference and reflection problems. Background noise was kept very low in the laboratory because no other machines were working during the experiments. Furthermore, background noise is effectively filtered from acoustic signal when the time synchronous averaged signal is used as will be explained in the next section.

\section{Signal Analysis}

The first step in signal analysis is usually to decompose the signal into a summation (or integral) of known functions. In Fourier analysis, for example, the signal is represented by the summation (or integral) of sinusoidal functions, the Fourier transform being the weight of each sinusoidal component. Likewise, wavelet analysis represents the signal as the summation (or integral) of given mother functions (mother wavelets) but, unlike Fourier transforms, it

Moreno-Sanchez, Ricardo $\quad$ February 17, 2011

10 
Comparison of Minimum Detectable Crack Size in a Geared System From Three Different Vibration Transducer Types

employs windows of variable dimensions, making it possible to represent local features as well as general trends. Large time intervals are used to analyze low frequency components and small intervals for high frequencies. The wavelet transform supplies the correlation coefficients between the signal and the mother wavelet according to scale and position [7].

As seen in Eq.(1), the wavelet coefficients, $C$, are defined as the time integral of the signal or function $f(t)$ multiplied by scaled and shifted versions of the mother wavelet $\Psi$. The resultant coefficient is, therefore, a function of the selected scale and position. Wavelets are particularly well suited for non stationary signals since the analysis can identify the precise time when an event occurs (specially low energy transients and discontinuities).

$$
C(\text { scale }, \text { position })=\int_{-\infty}^{+\infty} f(t) \psi(\text { scale }, \text { position }, t) d t
$$

It is important to select the right type of mother wavelet for the analysis at hand. In our case, a good recommendation would be to try to find a mother wavelet similar in shape to the expected signal generated by the mechanical fault that we are trying to detect. One such wavelet would yield large coefficients when the event occurs. Several studies confirm that Morlet wavelets have advantages when one tries to detect cracks in gears ([14], [15]).

The Hilbert transform is sometimes used in signal analysis. It is defined as the convolution between the signal $x(t)$ and $1 / t$ (see [28] and Eq. (2)), and it is intended to provide an estimate of the amplitude modulation in the signals or, in other words, to reveal a sketch of the low frequency events that modulate the signal.

$$
\hat{x(t)}=\frac{1}{\pi t} * x(t)=\frac{1}{\pi} \int_{-\infty}^{+\infty} \frac{x(\tau)}{(t-\tau)} d \tau
$$

Moreno-Sanchez, Ricardo $\quad$ February 17, 2011 


\section{Comparison of Minimum Detectable Crack Size in a Geared System From Three Different Vibration Transducer Types}

Alternatively, one may use the envelope signal, A(t), which corresponds to the modulus of the analytical signal, $\mathrm{z}(\mathrm{t})$, as in Eqs. (3) and (4).

$$
\begin{gathered}
z(t)=x(t)+j H[x(t)] \\
A(t)=|z(t)|
\end{gathered}
$$

Both, the wavelet transform and the Hilbert transform described above, are used in our signal analysis, but the first step is to get rid of non periodic events and to clean the noise as much as possible. This may be done by obtaining the Time Synchronous Average (TSA) of the signal which amounts to obtaining a "representative" rotation (average) using the raw signal from several rotations. As an example, Fig. 2 shows a raw signal (from the laser vibrometer in our test bench) during one rotation, as well as the TSA obtained using all revolutions in a 10 second period (since the shaft was rotating at $710 \mathrm{rpm}$, this TSA is a composite representation of 118 rotations). Most non-periodic events will be wiped out during averaging, whereas periodic events such as those produced by gear faults will still be present in the TSA [29]. For instance, it is very useful to eliminate the background noise in the microphone signal.

To illustrate how the Hilbert transform helps highlight (amplify) the modulating events at low frequencies, a gear with an incipient crack was installed in our test bench with a $75 \mathrm{Nm}$ loop torque and was forced to rotate at $710 \mathrm{rpm}(11.8 \mathrm{~Hz})$. The signal from the laser vibrometer was converted to a Time Synchronous Average and its spectrum plotted in Fig.

3a. The mesh frequency peak in this spectrum is located around $879 \mathrm{~Hz}$, but very little

Moreno-Sanchez, Ricardo $\quad$ February 17, 2011 
Comparison of Minimum Detectable Crack Size in a Geared System From Three Different Vibration Transducer Types
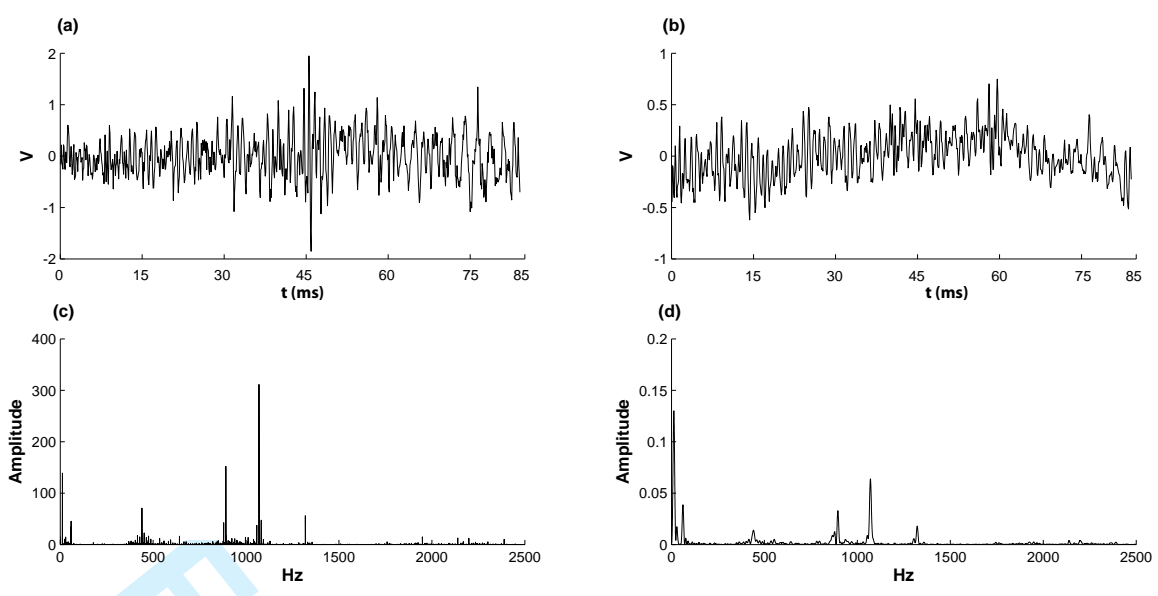

Figure 2: (a) Raw signal for one rotation. (b) Time Synchronous Average signal (TSA). (c) Frequency spectrum for raw signal. (d) Frequency spectrum for TSA.

information is obtained with respect to the defective tooth which should be "emitting" at the faulted gear rotation frequency, harmonics and sidebands. This modulation is easily found with the help of the Hilbert transform. Fig. 3b shows the spectrum of the Hilbert transform of the TSA signal. The high peaks in this spectrum correspond to motor axle rotation frequency (around 11.8Hz) and the faulted gear rotation frequency (around 14.6Hz).

The wavelet transform applied after the Hilbert transform will help bring up transient events and discontinuities. A sample comparison is shown in Fig. 4 where the wavelet coefficients are plotted (as a function of scale and time) for two crack sizes: 1mm (Fig. 4a) and $1.5 \mathrm{~mm}$ (Fig. 4b). It is possible to see how the larger crack size produces larger wavelet coefficients at those scales corresponding to the rotating frequency. Nevertheless, the increase in coefficients is much more significant when the Hilbert transform is applied prior to obtaining the wavelet transform. This can be seen in Figs. 4c and 4d which correspond to the wavelet coefficients of the Hilbert transform of the signals. Figure 4 is just

Moreno-Sanchez, Ricardo $\quad$ February 17, 2011 


\section{Comparison of Minimum Detectable Crack Size in a Geared System From Three Different Vibration Transducer Types}
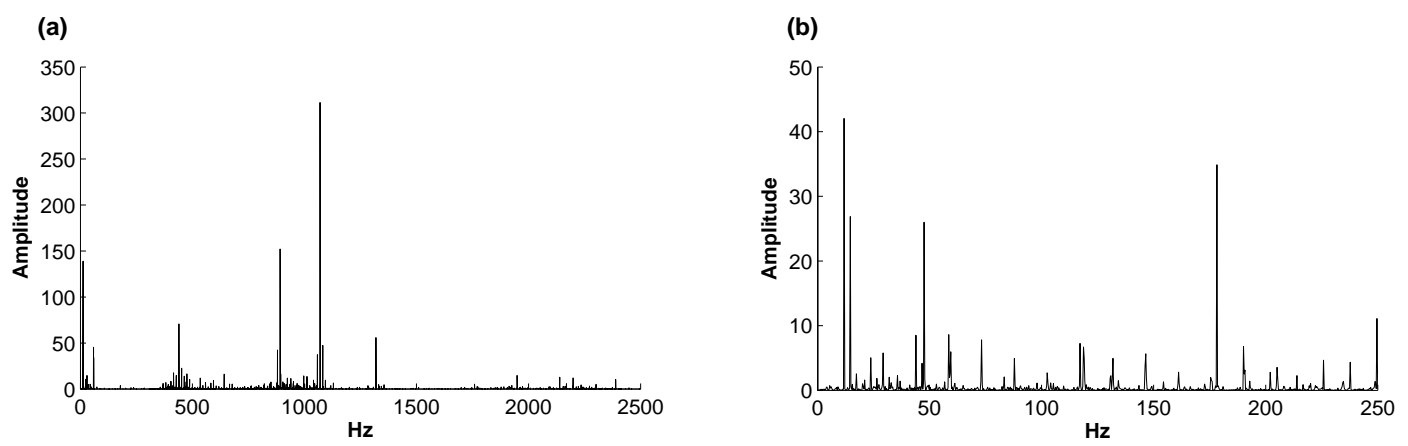

Figure 3: Test bench operating at 75Nm and 710rpm. (a) Frequency spectrum for TSA. (b) Frequency spectrum for TSA after Hilbert transform.

an example of the wavelet and Hilbert-wavelet values when the test bench operates at $75 \mathrm{Nm}$ and 710rpm, but it is worth emphasizing that these transforms were also obtained for all of the 144 experiments.

It should also be pointed out that the encoder signal allows us to determine the location of the defect with respect to an initially set reference angular position (time: $0.0506 \mathrm{~s}$ ).

Since, as has been mentioned, the defective tooth "emits" at the faulted gear rotation frequency, one should look for information in the wavelet transform at the scale corresponding to this rotation frequency. Our proposal is to evaluate the RMS of the wavelet coefficients at the aforementioned scale during one rotation. The end result of this analysis is a statistical parameter, that we called HWTSA (from Hilbert-Wavelet transform of Time Synchronous Averaged Signals) that helps quantify the defect size as will be shown in the next section. For the sample comparison shown in Fig. 4, the HWTSA is 0.47 for the $1 \mathrm{~mm}$ crack and 0.61 for the $1.5 \mathrm{~mm}$ of crack. Although this example seems to indicate that HWTSA increases with crack size, this hypothetical relationship will be validated using a multifactor experimental

Moreno-Sanchez, Ricardo $\quad$ February 17, 2011 
Comparison of Minimum Detectable Crack Size in a Geared System From Three Different Vibration Transducer Types
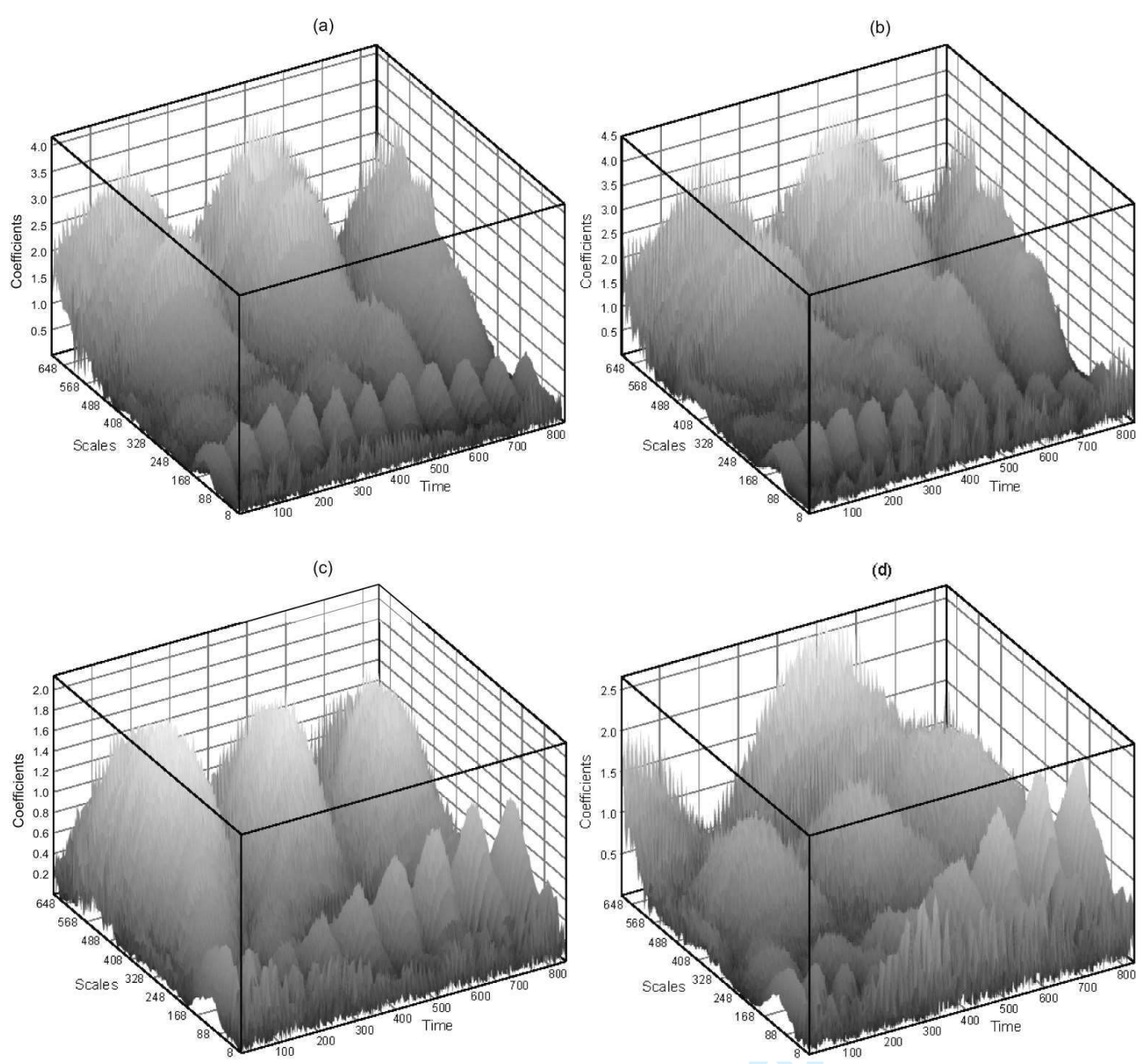

Figure 4: 3D wavelet transform coefficient plots. Test bench operating at $75 \mathrm{Nm}$ and $710 \mathrm{rpm}$.

Signal from the laser vibrometer. (a) Morlet wavelet transform with a $1 \mathrm{~mm}$ crack depth. (b) Morlet wavelet transform with a $1.5 \mathrm{~mm}$ crack depth. (c) Morlet wavelet after Hilbert transform with a $1 \mathrm{~mm}$ crack depth. (d) Morlet wavelet after Hilbert transform with a $1.5 \mathrm{~mm}$ crack depth. Time units: $10^{-4} \mathrm{~s}$ 
Comparison of Minimum Detectable Crack Size in a Geared System From Three Different Vibration Transducer Types

design.

Comparisons between HWTSA values for $1 \mathrm{~mm}$ and $1.5 \mathrm{~mm}$ cracks are shown in Table 2 (test bench operating at $75 \mathrm{Nm}$ and $710 \mathrm{rpm}$ ). Values obtained without the use of the Hilbert transform (WTSA) are also shown in the table. It can be seen that HWTSA is more sensitive to defect variations. Moreno et al ([30], [31]) have shown the advantages of using HWTSA over WTSA for detecting and classifying defects.

The signal processing described above may be carried out on signals generated by the accelerometer, as well as on signals generated by the microphone or the laser vibrometer. The general data processing scheme is depicted in Fig. 5.

As indicated above, the analytical signal is obtained after applying the Hilbert transform to the TSA (see Eqs. 3 and 4). Then, the Morlet wavelet transform is applied to the analytical signal obtaining the corresponding wavelet coefficients of Eq (5) which are, as shown in the equation, a function of scale and position.

$$
C(a, b)=\int_{0}^{T}|x(t)+j H[x(t)]| e^{-\frac{1}{2}\left(\frac{t-b}{a}\right)^{2}} e^{j 2 \pi * f_{c}\left(\frac{t-b}{a}\right)} d t
$$

Equation (5) is evaluated at the scale corresponding to the rotating frequency, which results in coefficients which are just a function of position and are evaluated for the entire

Table 2: Comparisons between HWTSA values for $1 \mathrm{~mm}$ and $1.5 \mathrm{~mm}$ cracks defects

\begin{tabular}{|c|c|c|c|}
\hline WTSA & HWTSA & WTSA & HWTSA \\
Crack 1mm & Crack 1mm & Crack 1.5mm & Crack 1.5mm \\
\hline 1.6304 & 0.4765 & 1.6558 & 0.6076 \\
\hline
\end{tabular}

Moreno-Sanchez, Ricardo $\quad$ February 17, 2011 
Comparison of Minimum Detectable Crack Size in a Geared System From Three Different Vibration Transducer Types

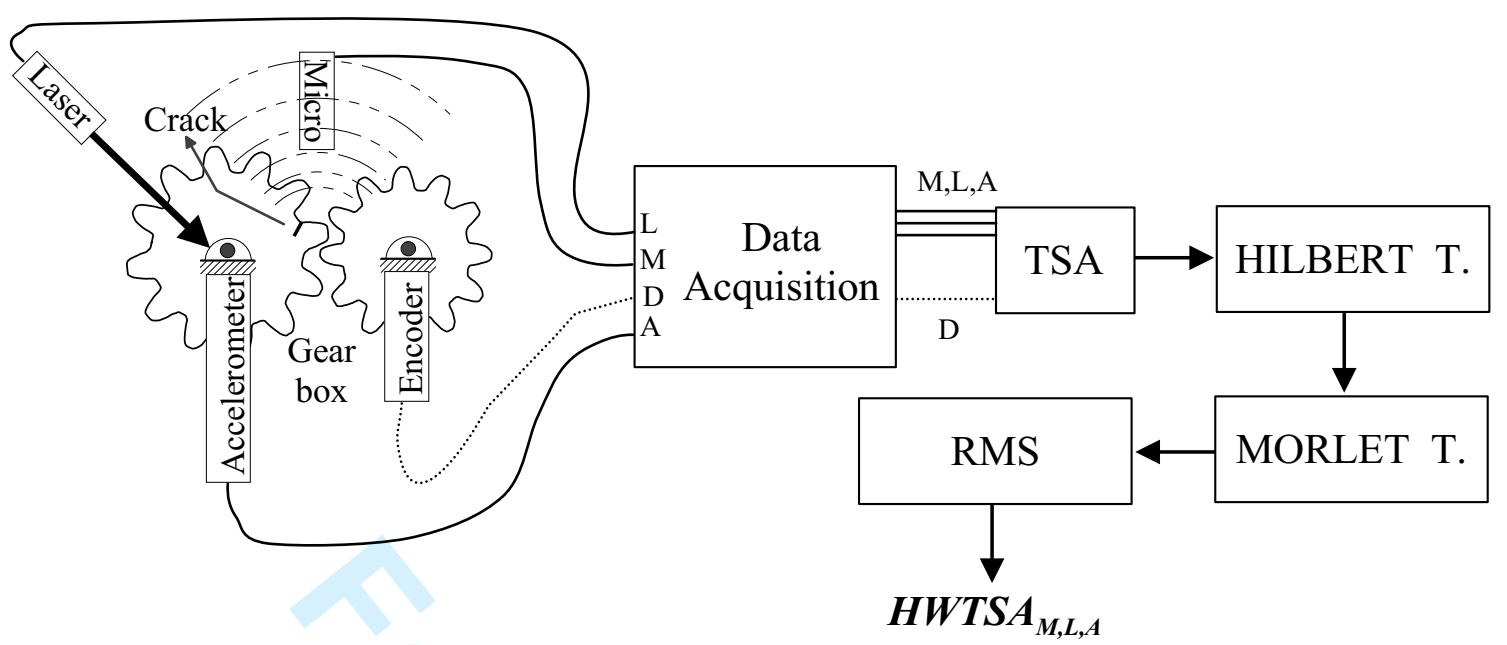

Figure 5: Signal processing for determining the HWTSA parameter.

(average) rotation. The HWTSA parameter is the RMS value of these coefficients:

$$
H W T S A=\left(\frac{1}{N} \int_{0}^{T}(C(b)-\overline{C(b)})^{2} d b\right)^{\frac{1}{2}}
$$

The relation between scale and the equivalent frequency (pseudo-frequency)[32] is given by the following expression:

$$
a=\frac{f_{c}}{f_{e q} T}
$$

Where a is the wavelet scale, Feq is the equivalent frequency $(\mathrm{Hz}), \mathrm{T}$ is the sampling pe$\operatorname{riod}(\mathrm{s} /$ ciclo), and $\mathrm{Fc}$ is the center frequency of a wavelet $(\mathrm{Hz})(\mathrm{Fc}=0.81$ for Morlet wavelets).

Table 3 shows the scale for every rotating frequency used in these experiments.

Moreno-Sanchez, Ricardo

February 17, 2011 
Comparison of Minimum Detectable Crack Size in a Geared System From Three Different Vibration Transducer Types

\section{Experimental Results}

A multifactor experimental design was conducted to determine the influence of the factors: torque, speed and defect size on the diagnosis variable HWTSA. Experiments were carried out for three levels of loop torque, four different rotational speeds and six defect sizes, and each combination of torque, speed and size was replicated twice resulting in a total of $144(3 \times 6 \times 4 \times 2)$ experiments. The number of replicates was estimated based on similar previous $2^{3}$ factorial design. This number of replicates is enough to evaluate the random variability (or "pure error") and the repeatability. Each experiment yielded a ten second long signal to be analyzed. Proper randomization of the experiments was employed looking for independence (no correlation between factors). The statistical significance of each factor on the HWTSA was assessed via an analysis of variance [22]. It is important to emphasize that the measurements from accelerometer, microphone and laser vibrometer were acquired simultaneously, so that the comparison between any two transducers is based on exactly the same physical phenomena.

The levels for each factor are specified in table 4, where a schematic of the damage inflicted to the gear is also shown. The cracks were simulated by cutting into the root of a tooth at 45 angle $(\alpha=45)$ for a variable depth $\mathrm{D}$ that determines the defect size. A cut differs from a fatigue crack in many respects, one of them being the thickness or opening which, in the case of the cut could not be made smaller than $0.7 \mathrm{~mm}$.

The analysis of variance decomposes the variability of HWTSA into contributions due to each factor having removed the effects of all others. The ANOVA table shows the results of the statistical tests conducted to determine which factors have a statistically significant

Moreno-Sanchez, Ricardo $\quad$ February 17, 2011 
Comparison of Minimum Detectable Crack Size in a Geared System From Three Different Vibration Transducer Types

Table 3: Wavelet scales corresponding to gear fault frequencies

\begin{tabular}{|c|c|c|c|}
\hline RPM & RPM & Pseudofreq & Wavelet \\
Motor & Faulted Gear & Faulted Gear & Scale \\
\hline 470 & 579 & 9.6 & 846 \\
\hline 590 & 727 & 12.1 & 670 \\
\hline 710 & 875 & 14.6 & 557 \\
\hline 830 & 1023 & 17 & 476 \\
\hline
\end{tabular}

Table 4: Factors and levels used for experimental design.

\begin{tabular}{|c|c|c|c|}
\hline Level & $\begin{array}{c}\text { Torque } \\
{[\mathrm{N} \cdot \mathrm{m}]}\end{array}$ & $\begin{array}{c}\text { Speed } \\
{[\mathrm{rpm}]}\end{array}$ & $\begin{array}{c}\text { Defect Size } \\
\text { [deep in } \mathrm{mm}])\end{array}$ \\
\hline 1 & 0 & 470 & 0.0 \\
\hline 2 & 75 & 590 & 0.5 \\
\hline 3 & 150 & 710 & 1.0 \\
\hline 4 & & 830 & 1.5 \\
\hline 5 & & & 2.0 \\
\hline 6 & & & 2.5 \\
\hline
\end{tabular}

Moreno-Sanchez, Ricardo $\quad$ February 17, 2011 
Comparison of Minimum Detectable Crack Size in a Geared System From Three Different Vibration Transducer Types

effect on HWTSA.

The parameters in the effect model for three factors are:

$$
H W T S A_{i j k l}=\mu+\tau_{i}+\beta_{j}+\gamma_{k}+\epsilon_{i j k l}
$$

Where $\tau_{i}$ is the defect effect in the ith level $(\mathrm{i}=1,6), \beta_{j}$ is the speed effect in the jth level $(\mathrm{j}=1,4), \gamma_{k}$ is the torque effect in the kth level $(\mathrm{i}=1,3), \epsilon_{i j k l}$ is the random effect.

Three factors are assumed to be fixed, treatment effects are deviations from the overall mean, so that $\sum \tau_{i}=0, \sum \beta_{j}=0$ and $\sum \gamma_{k}=0$. The ANOVA table decomposes the variability of HWTSA into contributions due to the three factors (defect, speed and torque) and a residual component which measures the variability amongst subjects at identical levels of the factors. Since Type III sums of squares is studied, the contribution of each factor is measured having removed the effects of all other factors. The P-values test the statistical significance of each of the factors. Since all 3 P-values are less than 0.05 , these factors have a statistically significant effect on HWTSA at the $95 \%$ confidence level.

Table 5 shows the results for our experiments when the microphone signal is used. The F-test is used to identify the significant factors [22], and since the P-values are all lower than 0.05, all three factors have a statistically significant effect on HWTSA at the $95 \%$ confidence level.

Figure 6 shows the mean values of HWTSA using the microphone for each factor at the $95 \%$ confidence level. Figs. 7 and 8 show the same information for accelerometer and laser, respectively.

The change in the mean values of HWTSA produced by a change in the levels of each

Moreno-Sanchez, Ricardo

February 17, 2011

20

Society for Experimental Mechanics, Inc. 
Comparison of Minimum Detectable Crack Size in a Geared System From Three Different Vibration Transducer Types

Table 5: ANOVA table for the microphone signal.

\begin{tabular}{|c|c|c|c|c|c|}
\hline FACTOR & Sum of Squares & Degree of Freedom & Mean Square & F-Ratio & P-Value \\
\hline Defect & 22.2915 & 5 & 4.45829 & 4.09 & 0.0017 \\
\hline Speed & 55.7279 & 3 & 18.576 & 17.03 & 0.0000 \\
\hline Torque & 34.8214 & 2 & 17.4107 & 15.96 & 0.0000 \\
\hline \hline RESIDUAL & 145.045 & 133 & 1.09056 & & \\
\hline TOTAL & 257.886 & 143 & & & \\
\hline
\end{tabular}
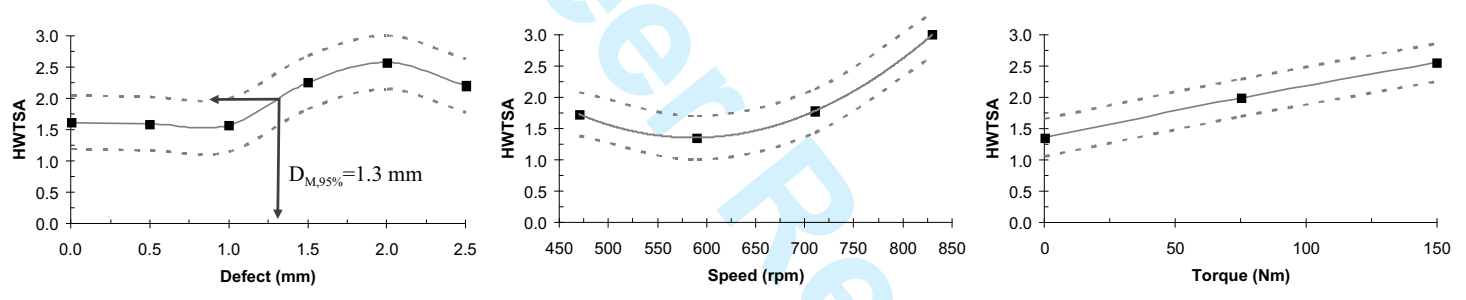

Figure 6: HWTSA behaviour with factors: defect, speed and torque. Microphone signal.
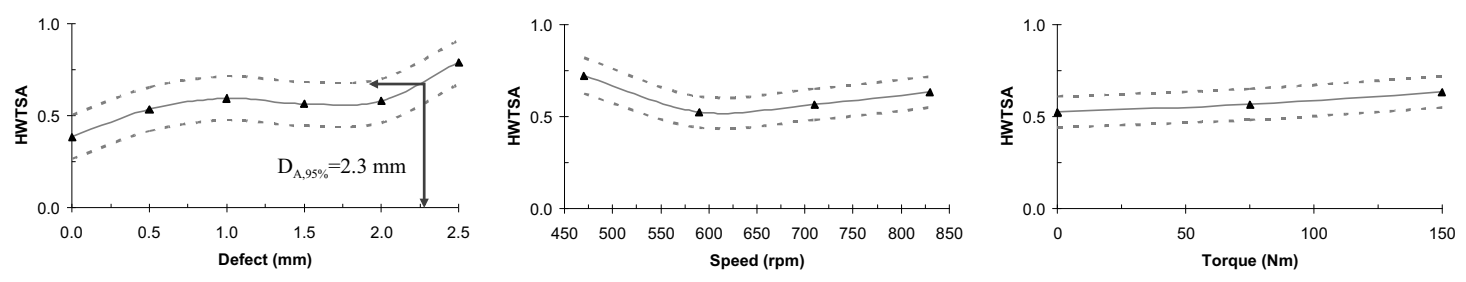

Figure 7: HWTSA behaviour with factors: defect, speed and torque. Accelerometer signal.

Moreno-Sanchez, Ricardo

February 17, 2011 
Comparison of Minimum Detectable Crack Size in a Geared System From Three Different Vibration Transducer Types

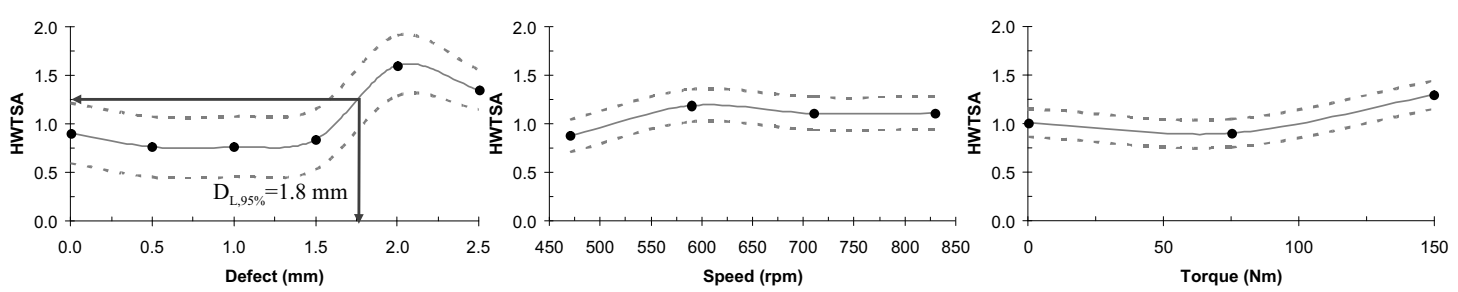

Figure 8: HWTSA behaviour with factors: defect, speed and torque. Laser vibrometer signal.

factor is frequently called the main effects because it shows the influence of the primary factors on the dependent variable.

Figures 6, 7 and 8 show that HWTSA for a gear without crack is lowest when the accelerometer signal is used, and largest (200\% larger) when the microphone signal is used, the value for the laser vibrometer signal being in between $(50 \%$ larger than in the accelerometer case).

Parameter HWTSA increases with defect size, but a threshold is reached from which HWTSA diminishes for increasing defect size. Similar conclusions have been reported by other authors [6] as well.

Least significant differences (LSD) was the method used for testing the statistically significant differences between means because the F-ratio was significant and comparisons were planned.

What LSD does is to form a confidence interval (using Student's t distribution) for each pair of means at the selected confidence level. The name of the method indicates that the magnitude of the limits represent the smallest difference between any two means that can be declared to represent a statistically significant difference. The method is recommended for

Moreno-Sanchez, Ricardo $\quad$ February 17, 2011 
Comparison of Minimum Detectable Crack Size in a Geared System From Three Different Vibration Transducer Types

the cases in which the F-test in the ANOVA table indicates significant differences amongst the sample means.

LSD intervals determine the confidence band around the average value shown in Figs. 6, 7 and 8 (having removed the effect from all other factors). These bands help us evaluate whether HWTSA is actually increasing due to progressive damage. In other words, the bands help us determine the minimum detectable crack size. These crack sizes are shown in the figures for the three sensors under consideration. The accelerometer begins to detect the crack when its depth is $D_{A, 95 \%}=2.3 \mathrm{~mm}$, which is probably too late since, under the testing conditions, the tooth breaks for a crack size of about 3mm. The Laser Doppler vibrometer begins to detect a crack when its size is $D_{L, 95 \%}=1.8 \mathrm{~mm}$, whereas the microphone shows the best crack detection capacity forcing HWTSA to change for a crack $1.3 \mathrm{~mm}$ deep.

Table 6 includes the minimum detectable crack size for each of the sensors, along with the rate of change of HWTSA with respect to size, speed and torque. The rate of change on HWTSA with respect to defect size is much higher than the rates of change with respect to torque and speed, regardless of the sensor used. The microphone has the best early detection capability, but the influence of torque and speed is also higher in this sensor. This means that a model than can separate or "understand" the effects of each factor to avoid false alarms is of greater importance when using a microphone as the monitoring sensor.

In general, the detection sensitivity is enhanced, and false alarms avoided, by characterizing the rate of change of HWTSA with respect to torque and speed in the operation range of the machine under consideration. In industrial applications this may be done by collecting data from the machine in good working conditions, and use this data to build up a history database with which to analyze the variation of HWTSA with operational factors.

Moreno-Sanchez, Ricardo $\quad$ February 17, 2011 


\section{Comparison of Minimum Detectable Crack Size in a Geared System From Three Different Vibration Transducer Types}

Other interesting result from factorial design is the interaction analysis between factors. This analysis lets us understand why it is possible that different researchers drew different conclusions when the comparisons between transducers were made at different torque and speed levels. "In some experiments it is possible to find that the difference in response between the levels of one factor is not the same at all levels of the other factors. When this occurs there is an interaction between factors". These ideas can be illustrated graphically by means of an interaction plot. These plots show the mean values of each level of Factor A response data for all levels of Factor B. The values are joined by lines so that lines approximately parallel indicate a lack of interaction between factor A and B [22].

Figure 9a shows the main values of HWTSA for each defect size level and each sensor. HWTSA values measured with microphone are higher than LDV every time. HWTSA measured with accelerometers has the lower values. Changes in HWTSA value are different depending of the defect levels for each transducer. If only two levels of defect size had been selected, different conclusions would have been obtained depending on which two levels were chosen. These are the reasons that made us select six levels in our experiments, sweeping a wide range before tooth break.

Figure 9b shows the transducer and speed interaction plots. It can be seen that the HWTSA value is more sensitive to speed changes when the microphone is used.

The presence of interactions suggests that, in order to select a transducer, it is necessary to take torque and velocity into account. For high torques and/or high speeds the microphone shows larger sensitivity than other transducers. For low torques and/or low speeds the HWTSA values yielded by the three transducers are similar.

The interaction plot between transducers and torque is shown in figure 9c. Clearly,

Moreno-Sanchez, Ricardo $\quad$ February 17, 2011 
Comparison of Minimum Detectable Crack Size in a Geared System From Three Different Vibration Transducer Types

Table 6: Minimum detectable crack size and rate of change of HWTSA with respect to size, torque and speed, for all three sensors.

\begin{tabular}{|c|c|c|c|c|}
\hline Sensor & $\begin{array}{c}l_{\text {min }} \text { Crack } \\
{[\mathrm{mm}]}\end{array}$ & $\begin{array}{c}\Delta \text { HWTSA } / \Delta \text { Defect } \\
{\left[\mathrm{mm}^{-1}\right]}\end{array}$ & $\begin{array}{c}\Delta \text { HWTSA } / \Delta \text { Torque } \\
{\left[\mathrm{Nm}^{-} 1\right]}\end{array}$ & $\begin{array}{c}\Delta \mathrm{HWTA} / \Delta \text { Speed } \\
{\left[\mathrm{rpm}^{-} 1\right]}\end{array}$ \\
\hline Microphone & 1.3 & 1.35 & 0.007 & 0.01 \\
\hline Laser & 1.8 & 1.52 & 0.005 & 0.0006 \\
\hline Accelerometer & 2.3 & 0.42 & 0.001 & 0.0006 \\
\hline
\end{tabular}

(a)

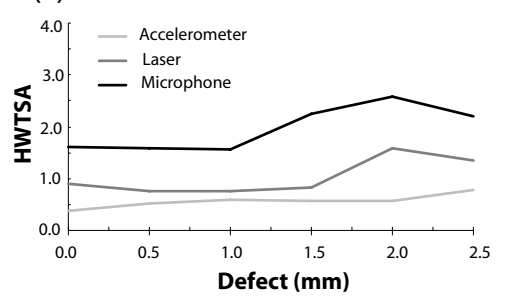

(b)

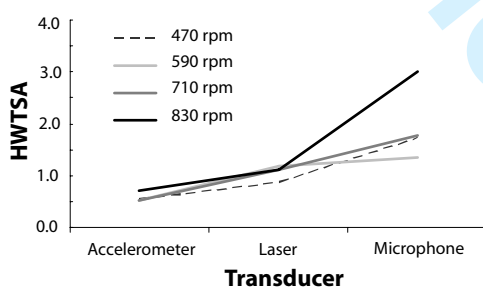

(c)

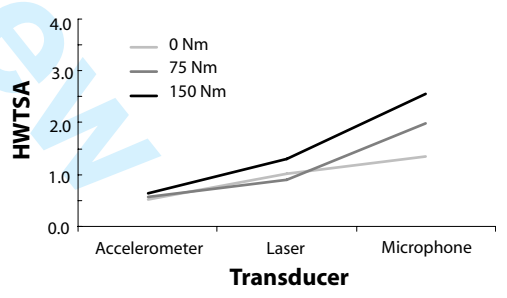

Figure 9: (a)Transducer and defect size interaction plot. (b)Transducer and speed interaction plot. (c)Transducer and torque interaction plot.

Moreno-Sanchez, Ricardo

February 17, 2011 
Comparison of Minimum Detectable Crack Size in a Geared System From Three Different Vibration Transducer Types

the interaction is large in this figure. Presence of interaction means that the difference in response between the levels of one factor is not the same at all levels of other factors. The interaction is low when lines in the interaction plot are parallel. For these reasons, 3 levels of torque are enough in this experiment (levels 2 and 3 of torque show almost parallel lines).

From the interaction plot one may conclude that different levels of torque and speeds must be analyzed to support the comparison between main effects. Otherwise, misleading conclusions may be drawn.

\section{Conclusions}

Multifactor experimental design has been proven useful to analyze the main effects of factors such as defect size, torque and speed on the dependent variable HWTSA obtained from signals of three different transducers. Experiments have been conducted in a close loop torque test rig and ANOVA tests have been performed to determine the statistical significance of each factor. The results were used to evaluate the main effects in the factorial experiments and to compare the minimum detectable crack size among all three sensors, which is a novel contribution of the work presented in this paper. All sensors were able to detect the crack, but the earliest detection was made with the microphone (when the crack was just $1.3 \mathrm{~mm}$ deep), followed by the laser vibrometer $(1.8 \mathrm{~mm}$ crack). The least sensitive sensor was, in this respect, the accelerometer $(2.3 \mathrm{~mm}$ crack). This systematic experimental design for comparing the LDV, microphone and accelerometer had not been undertaken in any previously published work.

Operational factors such as torque and speed have been included in the experiments to

Moreno-Sanchez, Ricardo

February 17, 2011

26

Society for Experimental Mechanics, Inc. 
Comparison of Minimum Detectable Crack Size in a Geared System From Three Different Vibration Transducer Types

quantify their effect on the detection process. It has been shown that the interactions of these factors on the monitoring variable (HWTSA) is quite significant. Comparisons between transducers carried out at constant torque and/or constant speed may yield very different conclusions depending on the level selected. This is the reason why a factorial experimental analysis was carried out sweeping a wide range of values for each factor (defect size, torque and speed) allowing us to estimate the main effects of each factor for several levels of the others, yielding conclusions that are valid over a range of experimental conditions. These interactions show that it is important to take the torque and speed into account when implementing a monitoring program.

\section{Acknowledgement}

The authors would like to show their gratitude to the "Fundacion Carolina" (Spain) and the "Universidad de Antioquia" (Colombia) for the support they have provided towards this investigation. The financial support of the Ministerio de Educacion y Ciencia (Spain), through project DPI2009-10230 "Optimizacion y Adaptabilidad de Suspensiones Neumaticas para Transporte y Maquinaria", is acknowledge and appreciated.

\section{References}

[1] Davis J.R., 2005, Gear materials, properties and manufacture, ASM International.

[2] Smith J.D., 2000, Gear Noise and Vibration, Ed Marcel Dekker Inc, New York.

Moreno-Sanchez, Ricardo $\quad$ February 17, 2011 
Comparison of Minimum Detectable Crack Size in a Geared System From Three Different Vibration Transducer Types

[3] Mobley, R, 1999, Root cause failure analysis, Butterworth-Heinemann. Massachusetts, USA.

[4] Decker H., 2002, "Crack Detection for Aerospace Quality Spur Gears", Technical Memorandum TM-2002-211492, ARL-TR-2682 NASA. Cleveland, Ohio.

[5] Keller J.A. and Grabill, P., 2003, "Vibration Monitoring of UH-60A Main Transmission Planetary Carrier Fault", The American Helicopter Society 59th Annual Forum, Phoenix, AZ.

[6] Mosher M., Pryor A. and Huff, E., 2001, "Evaluation of Standard Gear Metrics in Helicopter Flight Operation", Report 0289, NASA Ames Research Center. Moffett Field, CA.

[7] Newland D.E., 1994, An Introduction to Random Vibrations, Spectral and Wavelet Analysis, Prentice Hall. University of Cambridge.

[8] Loutridis S.J.,2008, "Self-Similarity in Vibration Time Series: Application to Gear Fault Diagnostics," ASME J. Vib. Acoust., 130(3), pp 1004-1013.

[9] Qin Y., Qin S. and Mao Y.,2008, "Research on Iterated Hilbert Transform and its Applications in Mechanical Fault Diagnosis," Mech. Syst. and Signal Pr. 22, pp 19671980.

[10] Lin J. and Zuo M., 2004, "Extraction of Periodic Components for Gearbox Diagnosis Combining Wavelet Filtering and Cyclostationary Analysis," ASME J. Vib. Acoust., 126(3), pp 449-452.

Moreno-Sanchez, Ricardo

February 17, 2011

28

Society for Experimental Mechanics, Inc. 
Comparison of Minimum Detectable Crack Size in a Geared System From Three Different Vibration Transducer Types

[11] Dellomo M.R., 1999, "Helicopter Gearbox Fault Detection: A Neural Network Based Approach," ASME J. Vib. Acoust., 121(3), pp 265-273.

[12] Forrester D., 1996, "Advanced Vibration Analysis Techniques for Fault Detection and Diagnosis in Geared Transmission Systems," PhD Thesis, Swinburne University of Technology.

[13] Wang W. and Wong A.K., 2002, "Autoregressive Model-Based Gear Fault Diagnosis," ASME J. Vib. Acoust., 124(2), pp 172-180.

[14] Lin J. and Qu L., 2000, "Feature Extraction Based on Morlet Wavelet and its Application for Mechanical Fault Diagnosis," J. Sound Vib., 234(1), pp 135-148.

[15] Zheng H., Li Z. and Cheng X., 2002, "Gear Fault Diagnosis Based on Continuos Wavelet Transform", Mech. Syst. and Signal Pr., 16(2-3), pp447-457.

[16] Moreno R., Pintado P., Alonso F.J., Chicharro J.M., Morales A.L. and Nieto, A.J., 2007, "Evaluacion y Comparacion de Modelos de Diagnostico de Fallos en Engranajes Utilizando las Senales de Vibraciones Mecanicas", $8^{\circ}$ Congreso Iberoamericano de Ingeniera Mecnica. Cusco, Peru.

[17] Tan Ch., Irving Ph. and Mba, D.,2007, "A Comparative Experimental Study on the Diagnostics and Prognostic Capabilities of Acoustic Emission, Vibration and Spectrometric Oil Analysis for Spur Gears," Mech. Syst. and Signal Pr.,21(6), pp 208-233.

[18] Baydar N. and Ball A., 2003, "Detection of Gear Faillures Via Vibration and Acoustic Signals Using Wavelet Transform, Mech. Syst. and Signal Pr.,17(4), pp 787-804.

Moreno-Sanchez, Ricardo $\quad$ February 17, 2011 
Comparison of Minimum Detectable Crack Size in a Geared System From Three Different Vibration Transducer Types

[19] Al-Ghamd A. and Mba D., 2006, "A Comparative Experimental Study on the Use of Acoustic Emission and Vibration Analysis for Bearing Defect Identification and Estimation of Defect Size", Mech. Syst. and Signal Pr.,20, pp 1537-1571.

[20] Shiroishi J. and Li Y., Kurfess, T., and Danyluk, S., 1997, "Bearing Condition Diagnostics Via Vibration and Acoustic Emission Measurements," Mech. Syst. and Signal Pr., 11(5) pp 693-705.

[21] Toutountzakis T., Keong Ch. and Mba D.,2005, "Application of Acoustic Emision to Seeded Gear Fault Detection," NDT\&E Int, 38 pp 27-36.

[22] Montgomery D., 2005, Design and Analysis of Experiments, 6th Edition, John Wiley and Sons, New York.

[23] Castellini P., Martarelli M. and Tomasini E.P., 2006, "Laser Doppler Vibrometry: Development of Advanced Solutions Answering to Technologys Needs", Mech. Syst. and Signal Pr., (20), pp 1265-1285.

[24] Nassif H., Gindy M. and Davis J., 2005, "Comparison of Laser Doppler Vibrometer With Contact Sensors for Monitoring Bridge Deflection and Vibration, NDT\&E International , 38, pp 213-218.

[25] Sung C., Tai H. and Chen C., 2000, "Locating Defects of a Gear System by Technique of Wavelet Transform, Mech. Mach. Theory, 35, pp 1168-1182.

[26] Heo G., Wang M.L. and Satpathi D., 1997, "Optimal Transducer Placement for Health Monitoring of Long Span Bridge", Soil Dynamics and Earthquake Eng., 16, pp 495-502.

Moreno-Sanchez, Ricardo $\quad$ February 17, 2011 
Comparison of Minimum Detectable Crack Size in a Geared System From Three Different Vibration Transducer Types

[27] Tatar K., Rantatalo M. and Gren P., 2007, "Laser Vibrometry Measurements of an Optically Smooth Rotating Spindle", Mechanical System and Signal Processing., 21, pp 1739-1745.

[28] Hanh S.L., 1996, Hilbert Transform in Signal Processing, Artech House, Boston.

[29] Lebold M., McClintic K., Campbell R., Byington C. and Maynard K., 2000, "Review of Vibration Analysis Methods for Gearbox Diagnostics and Prognostics", Proceedings of the 54th Meeting of the Society for Machinery Failure Prevention Technology, Virginia Beach, VA, pp623-634.

[30] Moreno R., Pintado P., Chicharro J.M., Morales A.L., Nieto A.J. and Alonso F.J., 2008, "Deteccion de Fallos Incipientes en Engranajes Utilizando las Transformadas HilbertWavelet en el Analisis de Vibraciones Mecanicas", XVII Cong. Nal. Ing. Mec., pp 11091117.

[31] Moreno R., Pintado P., Chicharro J.M., Morales A.L. and Nieto A.J., 2009, "Methodology for Evaluating Neural Networks Inputs for Incipient Fault Detection in Gears", Proceedings of the 2009 IEEE Int. Conf. on Mechatronics, Spain, paper 4049.

[32] Abry P., 1997, Ondolettes et Turbulence. Multiresolutions, Algorithmes de Decomposition, Invariance Dchelles, Ed Diderot, Paris.

Ricardo Moreno is a full time lecturer of Mechanical Engineering at University of Antioquia (Medellin, Colombia). He received his B.Sc. degree in Mechanical Engineering Moreno-Sanchez, Ricardo $\quad$ February 17, 2011 31 
Comparison of Minimum Detectable Crack Size in a Geared System From Three Different Vibration Transducer Types

from National University of Colombia and a M.Sc. degree in Engineering from University of Antioquia. His research interests include Mechatronics, Signal Processing and Mechanical Design. He has worked for BMW (Munich, Germany) and Kawasaki Heavy Industries (NishiAkashi, Japan). He was a visiting scholar at MIT (Cambridge, USA), as a part of the Precision Engineering Research Group (PERG). He is currently studying a fourth year of the Ph.D program in Mechatronics at University of Castilla La Mancha (Ciudad Real, Spain).

Jose Manuel Chicharro is associate professor of Mechanical Engineering at the University of Castilla - La Mancha (Ciudad Real, Spain). He received his Engineer degree in 1994 and the Ph. D. degree from Universidad Politcnica de Madrid (Spain) in 2000. His research interests include Metrology, Mechatronics, Vibrations and Mechanical Design. He has published twenty articles, four books and several educational publications. He has worked as consultant for industrial mechanical projects in many occasions.

Publio Pintado graduated as a Mechanical Engineer from the University of Seville (Spain) in 1986 and obtained his Ph.D. from the same university in 1989. He was a Fulbright Fellow in the Virginia Polytechnic Institute during two academic years (1989-1991). He has been Associate Professor with the University of Seville (1991-1998), and he is currently a Full Professor of Mechanical Engineering at the University of Castilla La Mancha (Spain). His research interests lie along two lines: vibrations and mechanism design. With respect to vibrations he works on road damage caused by heavy vehicle suspensions, active control of pneumatic suspensions, and diagnosis of machinery. With respect to mechanism design he works on the design of staircase-climbing wheelchairs. He has published forty articles and two books, is author of two patents in Spain, and one commercial computer application. He has worked as consultant for private enterprises in many occasions.

Moreno-Sanchez, Ricardo $\quad$ February 17, 2011 
Comparison of Minimum Detectable Crack Size in a Geared System From Three Different Vibration Transducer Types

\section{Tables}

Table 1. Sensitivities and frequency responses of transducers.

Table 3. Wavelet scales corresponding to gear fault frequencies.

Table 2. Comparisons between HWTSA values for $1 \mathrm{~mm}$ and $1.5 \mathrm{~mm}$ cracks defects.

Table 4. Factors and levels used for experimental design.

Table 5. ANOVA table for the microphone signal.

Table 6. Minimum detectable crack size and rate of change of HWTSA with respect to size, torque and speed,for all three sensors. 
Comparison of Minimum Detectable Crack Size in a Geared System From Three Different Vibration Transducer Types

\section{List of Figure Captions}

Figure 1. Test bench. 1-Laser Doppler vibrometer. 2-Microphone. 3-Accelerometer. 4Adjustable torque coupling. 5-Encoder. 6-Speed regulator. 7- Data acquisition hardware. 8- Spur gears. 9-Laser point location.

Figure 2. (a) Raw signal for one rotation. (b) Time Synchronous Average signal (TSA). (c) Frequency spectrum for raw signal. (d) Frequency spectrum for TSA.

Figure 3. Test bench operating at 75Nm and 710rpm. (a) Frequency spectrum for TSA. (b) Frequency spectrum for TSA after Hilbert transform.

Figure 4. 3D wavelet transform coefficient plots. Test bench operating at $75 \mathrm{Nm}$ and $710 \mathrm{rpm}$. Signal from the laser vibrometer. (a) Morlet wavelet transform with a $1 \mathrm{~mm}$ crack depth. (b) Morlet wavelet transform with a $1.5 \mathrm{~mm}$ crack depth. (c) Morlet wavelet after Hilbert transform with a 1mm crack depth. (d) Morlet wavelet after Hilbert transform with a $1.5 \mathrm{~mm}$ crack depth.

Figure 5. Signal processing for determining the HWTSA parameter.

Figure 6. HWTSA behaviour with factors: defect, speed and torque. Accelerometer signal. Figure 7. HWTSA behaviour with factors: defect, speed and torque. Microphone signal.

Figure 8. HWTSA behaviour with factors: defect, speed and torque. Laser vibrometer signal.

Figure 9. (a)Transducer and defect size interaction plot. (b)Transducer and speed interaction plot. (c)Transducer and torque interaction plot.

Moreno-Sanchez, Ricardo $\quad$ February 17, 2011 